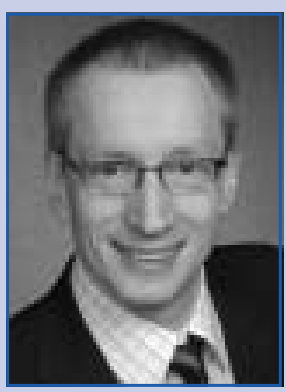

Prof. Dr. Andreas Hoffjan ist Inhaber des Lehrstuhls Unternehmensrechnung und Controlling an der Technischen Universität Dortmund sowie Mitherausgeber der Zeitschrift Controlling.

\title{
Was bedeutet die Regulierungswut für das Controlling?
}

Andreas Hoffjan

Ob beim umstrittenen Fahrdienst Uber oder bei der Diskussion um den StromKapazitätsmarkt, keine Woche scheint zu vergehen, ohne dass aus Berlin oder Brüssel ein neues Bürokratiemonster kommt. Kann das Controlling helfen, das Regulierungskorsett aufzuschnüren? Wächst dem Controller innerbetrieblich mit dem Regulierungsmanagement ein Wettbewerber um die Datenhoheit heran? Oder bleibt am Ende des Tages doch nur Mehrarbeit? Am Beispiel zweier regulierter Branchen wird nachfolgend aufgezeigt, welche Antworten das Controlling geben kann.

Regulierungsmanagement heißt das neue Zauberwort oder die unternehmerische Antwort auf eine hohe Regulierungsdichte. Was wird darunter verstanden? Regulierungsmanagement bezeichnet allgemein die Führung und Anpassung eines Unternehmens im Hinblick auf den Umgang mit administrativ regulierenden Eingriffen (vgl. Büdenbender/Rosin, 2005, S. 12 ff.). In der praktischen Umsetzung wird diese Schnittstellenfunktion häufig in operatives und strategisches Regulierungsmanagement unterschieden (vgl. PricewaterhouseCoopers, 2008, S. 306 ff. und S. $311 \mathrm{ff}$.). Ersteres dient vornehmlich der Ausgestaltung des Informationssystems nach regulatorischen Vorgaben. Insbesondere das interne Daten- und Informationsmanagement stehen hier im Vordergrund. Beim strategischen Regulierungsmanagement geht es um die Entscheidungsunterstützung bei der Strategiefindung, die Identifikation von Chancen und Risiken und die Erstellung eines übergreifenden Handlungsrahmens (vgl. Ehlers/Wernau, 2008, S. 70). Insgesamt obliegt dem Regulierungsmanagement die Zusammenstellung der notwendigen Informationen im Hinblick auf die Vorgaben der Genehmigungsbehörde und die optimale betriebswirtschaftliche Anpassung des Unternehmens an das Regulierungsumfeld (vgl. Landgrebe, 2006, S. 2).
Die verwandten Aufgaben unterstreichen bereits die inhaltliche Nähe von Controlling und Regulierungsmanagement. Mit zunehmender Regulierungsintensität dürfte der Einfluss des Regulierungsmanagements auf die Unternehmenssteuerung weiter zunehmen. Wird dann möglicherweise das klassische Controlling bei entgeltregulierten Unternehmen abgelöst? Welche Aufgaben muss ein Controller beherrschen, um seine Steuerungsverantwortung zu behaupten? Wie kann konkret die Zusammenarbeit zwischen den kaufmännischen Funktionen Regulierungsmanagement und Controlling aussehen?

In einer Studie zum Regulierungsmanagement in der Energiewirtschaft konnten die in Abb. 1 dargestellten vier Formen der Zusammenarbeit identifiziert werden (vgl. Schuchardt et al., 2015). Dabei wird das mit dem Werterhalt der vorhandenen Infrastruktur und Anlagen betraute Asset Management (AM) durchgängig als der technische Part im Planungsprozess angesehen. Die verschiedenen Ausprägungen der Zusammenarbeit machen sehr gut den konkurrierenden Einfluss auf die Unternehmenssteuerung deutlich.

Im übergeordneten Regulierungsmanagement werden weite Teile der kaufmännischen Steuerung übernommen. Der Einfluss des Regulierungsmanagements auf die kaufmännische Unternehmenssteuerung ist hierbei am höchsten, da alle entscheidungsrelevanten Daten in dieser Abteilung gebündelt werden. Das Controlling hingegen ist nicht am Abstimmungsprozess beteiligt und kann nur schwer Optimierungen in von Regulierung betroffenen Bereichen anstoßen.

Im Rahmen des koordinativen Regulierungsmanagements bilden Asset Management und Controlling zwei unabhängige Säulen, koordiniert vom Regulierungsmanager. Damit soll eine unabhän- 
gige Instanz mögliche Konflikte zwischen kaufmännischen und technischen Anforderungen auflösen und die Funktion des Schnittstellenmanagements leben. Bezüglich der Investitionsplanung wird beispielsweise im Controlling und Asset Management jeweils eine Priorisierung der wichtigsten Investitionsmaßnahmen erstellt. Diese werden im Regulierungsmanagement abgeglichen, um die größtmögliche Schnittmenge zu ermitteln.

Beim controllingintegrierten Regulierungsmanagement obliegt die Zusammenführung der Informationen dem Controlling, während das Regulierungsmanagement einen Großteil der strategischen Aufgaben übernimmt. Im Controlling werden schließlich die finalen (Investitions-)Entscheidungen getroffen. Die Bereitstellung entscheidungsrelevanter Informationen unter Berücksichtigung des regulatorischen Umfeldes stellt demnach die Kernaufgabe des Regulierungsmanagements dar. Der Einfluss des Controllings auf die Unternehmensführung ist höher als in den ersten beiden Ausprägungen.

In der vierten Organisationsform übernimmt das kommunikative Regulierungsmanagement die operativen Aufgaben und bildet damit vornehmlich die Kommunikationsfunktion zur Regulierungsbehörde ab. Die strategischen Aufgaben des Regulierungsmanagements liegen in diesem Fall beim Controlling oder außerhalb des Netzbetreibers. Der Einfluss des Controllings auf die kaufmännische Unternehmenssteuerung ist somit sehr hoch. Eine ausschließliche Fokussierung auf operative Aufgaben ist in der Praxis jedoch kaum noch anzutreffen. Regulierungsmanagement umfasst inzwischen mehr als eine solche „Briefträgerfunktion", was auch dem Controller bewusst sein sollte.

Unabhängig von der konkreten Ausgestaltungsform sind eine abteilungsübergreifende Zusammenarbeit und die $\mathrm{Ab}$ bildung des regulatorischen Einflusses auf die kaufmännische Steuerung erfolgskritisch. Umso wichtiger wird es, dass das Controlling regulatorische Rahmenbedingungen in seine Planungen integriert.

Auf welche Arbeiten sollte sich das Controlling mit zunehmender Regulierungsintensität einstellen? Insbesondere im Rahmen der Entgeltregulierung sind die

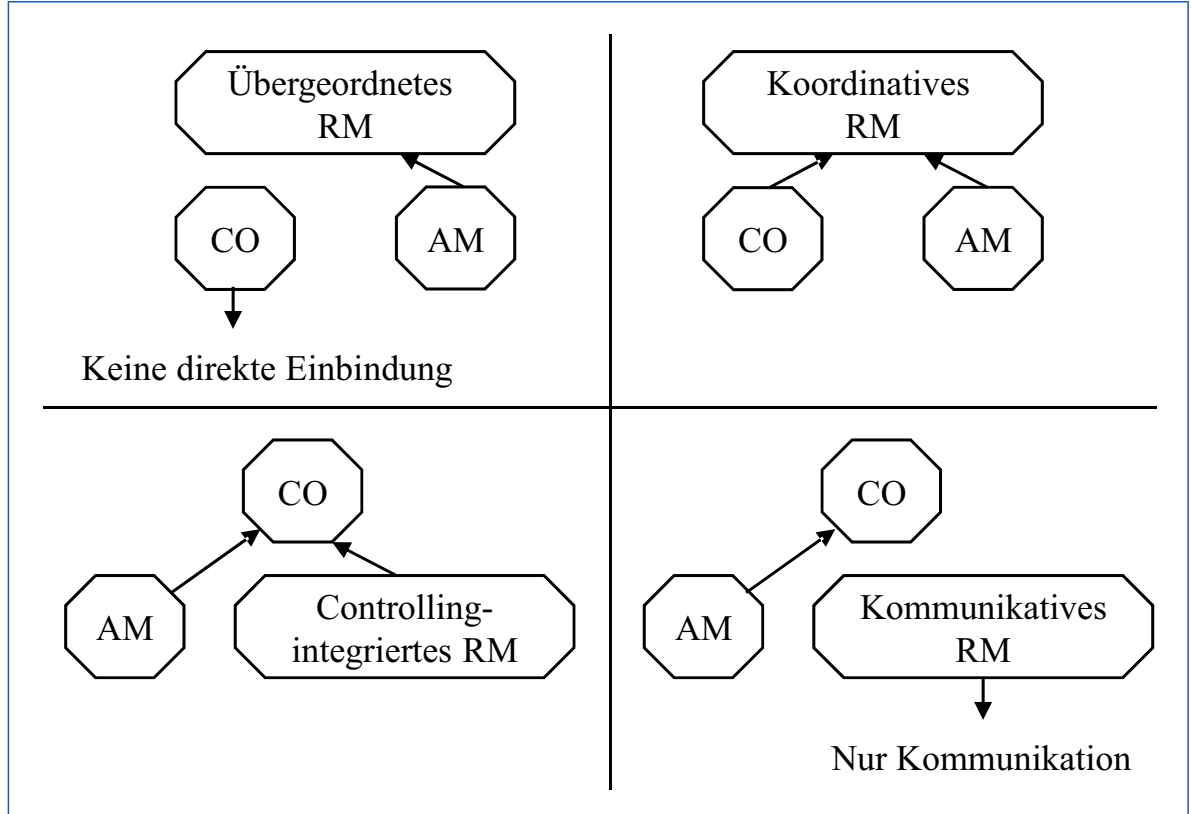

Abb. 1: Zusammenarbeit Controlling (CO) und Regulierungsmanagement (RM) (vgl. Schuchardt et al., 2015)

Unternehmen verpflichtet, der Regulierungsbehörde einen detaillierten Einblick in die Inhalte und Methodik der Kostenund Preiskalkulation zu gewähren. Um diesem Anspruch gerecht zu werden, ist es für die Unternehmen erforderlich, einen umfangreichen Bestand an Informationen aufzubauen und zu pflegen. Da mitunter ein Teil der von den Regulierungsbehörden geforderten Informationen nicht unmittelbar im Rechnungswesen vorhanden ist, ergeben sich zusätzliche Anforderungen an das Controlling und das Informationssystem der Unternehmen. Ein zu diesem Zweck erweitertes Informationssystem kann bei den betroffenen Unternehmen zusätzliche Kosten verursachen: zum einen unmittelbar und direkt durch den Einsatz zusätzlicher Ressourcen (Angestellte, IT-Kapazität, etc.), zum anderen aber auch indirekt in Form von Komplexitätskosten durch die gestiegenen Anforderungen an die Informationsverarbeitung und Entscheidungsvorbereitung.

In welchem Maße der Umfang des Informationssystems aufgrund regulativer Maßnahmen zunimmt, ist sowohl von der Menge der geforderten Einzelinformationen als auch von der seitens der Regulierungs- oder Kontrollbehörde vorgeschriebenen Qualität der Informationen abhängig. Eventuell ergeben sich durch die Regulierung bzw. deren Kontrolle auch neue Anforderungen an den Prozess der Informationssammlung und -verarbeitung sowie die Art der Ergebnisdarstellung.

Das Controlling in entgeltregulierten Unternehmen unterscheidet sich somit deutlich von dem anderer Branchen, unter anderem weil es einen zusätzlichen Adressatenkreis gibt, der bei der Gestaltung des Informationssystems, aber auch im Planungs- und Kontrollsystem berücksichtigt werden muss.

Die konkrete Umsetzung sei am Beispiel europäischer Großflughäfen aufgeführt (vgl. Endenich et al., 2014). Denkbar ist hier sowohl die Einführung und Einbindung kompletter regulatorischer Parallelbestehender Rechnungen durch regulatorisch geforderte Datensichten (vgl. Pedell, 2008, S. 684). Wie die Ergebnisse der empirischen Studie zeigen, ist die Mehrheit der Flughäfen aus Effizienzgründen bestrebt, eine Konsistenz zwischen dem allgemeinen Rechnungswesen und speziellen Berechnungen für Entgeltzwecke herzustellen. Eine integrierte interne Erlösund Kostenrechnung ist vor allem für Flughäfen mit geringer Regulierungsintensität das Rechenwerk, aus dem sie direkt die Zahlen für die Entgeltberechnung bereitstellen. Flughäfen mit mittlerer Regulierungsintensität versuchen, ihr internes Rechnungswesen den regulatorischen Vorgaben anzupassen. Konkret nehmen $11 \%$ der befragten Flughäfen rechnungen als auch nur die Ergänzung 
für Entgeltzwecke Adaptionen in der Kostenrechnung vor.

Ca. ein Drittel der Flughäfen, insbesondere solche unter hohem Regulierungseinfluss, erstellen eine komplette regulatorische Parallelrechnung. Die geforderte Entgeltstruktur stimmt meistens nicht mit der Organisationsstruktur überein. Für die regulierten Entgelte ist dann eine separate Hierarchie im SAP-System anzulegen, um die Kostenallokation sauber darzustellen. Typischerweise ist die Kostenallokation der größte Unterschied zwischen interner und regulatorischer Kostenrechnung. Dies betrifft die beiden Geschäftsbereiche Aviation, also alle Aktivitäten zur Abfertigung von Flügen, deren Passagiere und Fracht, sowie den Bereich Non-Aviation, in dem alle übrigen Aktivitäten des Flughafenbetreibers (Geschäfte, Parken) zusammengefasst sind. Die Kostenverteilung erfolgt anhand von Flächen, z. B. Verkehrswegeflächen, und ist ein kontroverses Thema mit Fluggesellschaften und Regulierern. Die Umsetzung der Zusatzrechnungen erzeugt bei den Flughäfen zum Teil erheblichen manuellen Aufwand. Im Ergebnis korreliert der Mehraufwand in der Kosten- und Erlösrechnung positiv mit der Strenge des
Regulierungsregimes. Es ist eine der Aufgaben des Controlling, diesen Aufwand zu begrenzen, indem es die Unterschiede zwischen interner, regulatorischer und externer Rechnungslegung auf das notwendige Maß beschränkt, um eine möglichst gute Kongruenz zwischen den Rechenwerken herzustellen.

Die ausufernde Regulierung erweitert das Aufgabenspektrum des Controllers. Sein Sachverstand ist gefordert, um die administrative Komplexität in effizienten innerbetrieblichen Strukturen abzubilden, idealerweise in Form integrierter Rechnungen. Für betriebliche Entscheidungen muss er die Auswirkungen des regulatorischen Rahmens auf die finanziellen Steuerungsgrößen abbilden können, sonst verliert er seine Transparenzverantwortung an den Regulierungsmanager. Ein um regulatorische Erfordernisse erweitertes Blickfeld macht seine Arbeit auf jeden Fall zukunftsfester, verheißt doch die politische Großwetterlage noch mehr Regulierung. Ein guter Controller wird die „dunklen Wolken“ am Reg(ul)ierungshimmel schon jetzt ausmachen können.

\section{Literatur}

Büdenbender, U./Rosin, P., Zuverlässigkeit von Maßnahmen des Regulierungsmanagements vor dem Hintergrund der Entflechtungsvorgaben des neuen Energiewirtschaftsgesetzes, Berlin 2005.

Ehlers, M./Wernau, O., Umsetzungsmöglichkeiten des Regulierungsmanagements, in: Energiewirtschaftliche Tagesfragen, 58. Jg. (2008), H. 12, S. 70-72.

Endenich, C./Brandau, M./Hoffjan, A./Müller, F., Zum Einfluss der Entgeltregulierung auf die Unternehmenssteuerung - Eine empirische Analyse europäischer Großflughäfen, in: Zeitschrift für betriebswirtschaftliche Forschung, 66. Jg. (2014), H. 3, S. 242-268.

Landgrebe, J., Liberalisierung und Regulierungsmanagement im Telekommunikationsmarkt, Wiesbaden 2006.

Pedell, B., Controlling unter dem Einfluss regulatorischer Vorgaben, in: Controlling, 20. Jg. (2008), S. 681-688.

PricewaterhouseCoopers, Entflechtung und Regulierung in der deutschen Energiewirtschaft: Praxishandbuch zum Energiewirtschaftsgesetz, 2. Aufl., Freiburg 2008.

Schuchardt, L./Finger, H./Hoffjan, A., Strategisches Regulierungsmanagement in der anreizregulierten Netzwirtschaft, erscheint in: Zeitschrift für betriebswirtschaftliche Forschung, 67. Jg. (2015), H. 1. 